\title{
Efficacy of Timothy grass allergy immunotherapy tablet (AIT) treatment in Canadian children and adults with grass pollen-induced allergic rhinoconjunctivitis (ARC)
}

\author{
Jacques Hebert ${ }^{*}$, Hendrik Nolte ${ }^{2}$, Jennifer Maloney ${ }^{2}$, Michael Blaiss $^{3}$, Harold S Nelson $^{4}$ \\ From Canadian Society of Allergy and Clinical Immunology Annual Scientific Meeting 2011 \\ Quebec, Canada. 20-23 October 2011
}

\section{Background}

The effect of Timothy grass AIT treatment on a Canadian subpopulation was assessed post-hoc using data from 2 randomized, double-blind, trials designed to evaluate grass AIT in North American subjects.

\section{Methods}

Subjects 5 years and older with ARC with/without asthma received once-daily 2800 BAU sublingual grass AIT (oral lyophilisate, Phleum pratense, 75,000 SQ-T, $\sim 15 \mu \mathrm{g}$ Phl p5) or placebo starting approximately 16 weeks before and continuing throughout the 2009 grass pollen season (GPS). Subjects used daily e-diaries to record ARC symptoms and use of symptomatic medications from randomization through study end (approximately 24 weeks). The primary efficacy endpoint comprised the average total combined daily symptom and medication score (TCS) during the entire GPS. Secondary endpoints included average daily symptom score (DSS) and average daily medication score (DMS). Safety was assessed by monitoring adverse events (AEs).

\section{Results}

The Canadian subpopulation included 103 subjects (46 pediatric [5-17y]; 57 adult [18-65y]). AIT-treated subjects showed reductions, vs placebo, of $38 \%(P=.016)$ for entire and $45 \%(P=.005)$ for peak seasons in TCS; of $38 \%$ $(P=.013)$ for entire and $40 \%(P=.008)$ for peak seasons in DSS; and of $39 \%$ for entire $(P=.238)$ and $60 \%(P=.005)$ for peak seasons in DMS. Among the overall population, most treatment-related AEs were mild or moderate. No serious or life-threatening treatment-related AEs occurred; no new safety concerns emerged.

\section{Conclusions}

Timothy grass AIT, a novel therapeutic modality, significantly improved ARC caused by Timothy grass pollen and related grasses in Canadian adults and children 5 years and older.

\section{Author details}

${ }^{1}$ Centre de Recherche Appliquee en Allergie de Quebec, Quebec, Canada. ${ }^{2}$ Merck \& Co., Kenilworth NJ, USA. ${ }^{3}$ Departments of Pediatrics and Medicine, University of Tennessee Health Science Center, Memphis TN, USA.

${ }^{4}$ Department of Medicine, Division of Allergy and Immunology, National Jewish Health, Denver CO, USA.

Published: 14 November 2011

\section{doi:10.1186/1710-1492-7-S2-A17}

Cite this article as: Hebert et al:: Efficacy of Timothy grass allergy immunotherapy tablet (AIT) treatment in Canadian children and adults with grass pollen-induced allergic rhinoconjunctivitis (ARC). Allergy, Asthma \& Clinical Immunology 2011 7(Suppl 2):A17. 